\title{
Control of Deformation of Elastic Polymer-Abrasive Circles at their Wear
}

\author{
Dmitriy Podashev
}

Irkutsk National Research Technical University, 83 Lermontov street, 664074, Irkutsk, Russia

dbp90@mail.ru

\begin{abstract}
Keywords: Elastic Polymer-Abrasive Circle, Wear of Instrument, Deformation Of Instrument, Deformation Regulation
\end{abstract}

\begin{abstract}
The article is devoted to the study of the wear intensity of elastic polymer-abrasive circles when processing the surfaces of parts made of high-strength aluminum alloys. Empirical dependencies of wear on cutting speed and tool deformation are obtained, on the basis of which method of tool deformation correction with long-term, continuous surface treatment is proposed. In practice, such a procedure is necessary due to the loss of process performance that occurs due to the reduction of tool deformation due to wear. The proposed technique allows to effectively control the finishing process as the tool is worn out.
\end{abstract}

\section{Introduction}

The problem of mechanization and automation of manual labor during finishing cleaning and finishing operations is relevant for modern mechanical engineering. For example, in the aviation industry, the design of any aircraft includes more than 50 large and long parts on which these operations are required. These parts are manufactured, usually by milling. Many works are devoted to the research of the milling process and the assurance of the quality of the surface layer of parts in this type of machining, for example $[1,2,3]$. However, even with a well-organized technology for manufacturing parts by milling, including on CNC machines, the surface roughness required in the drawing is not provided at the transition points when changing the direction of feeding, when processing curved surfaces, random defects associated with the actual state of the cutter, etc., also regularly occur.

Today, most finishing cleaning and finishing operations are performed manually, while the proportion of labor intensity of manual work on the finishing process of such parts can reach $65 \%$ of the total labor intensity of manufacturing parts after milling.

Machining methods, such as grinding, polishing, volumetric vibration machining, etc., are very effective ways to solve the problem of surface grinding. The works of many scientific teams are devoted to the study of these abrasive processing methods, for example $[4,5,6,7]$.

Each of these abrasive methods can be characterized by a different level of efficiency. Each method had certain property and disadvantages, as well as areas of effective application.

It should be noted that when machining with rigid tools, it is difficult to clear a thin surface layer (especially aluminum alloy parts widely used in the aircraft industry) due to the possibility of removing a certain layer of material and violating the required accuracy in size.

Volumetric vibration machining methods were very effective and were used for finishing metal parts with dimensions of up to $300 \mathrm{~mm}$. However, for the processing of large and long parts, examples of which are given in Figure 1, the use of these methods is not economically feasible, since large and expensive equipment, as well as a large amount of preparatory and final work, are necessary for their implementation. 
In connection with the above, the most promising direction capable of effectively solving these problems in ensuring the quality of finishing of large-sized, complex-shaped and long-sized parts taking into account the peculiarities of their sizes and structures is the treatment with polymerabrasive circles with a bond of nonwoven materials that have high elasticity.

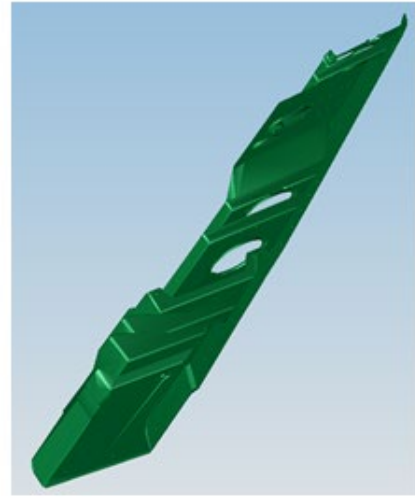

a)

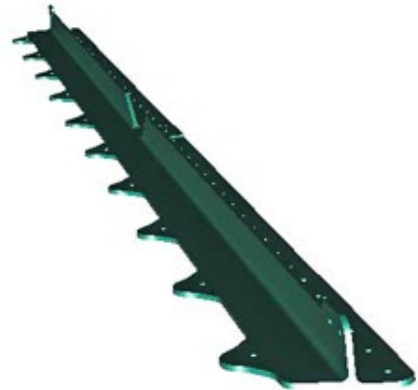

b)

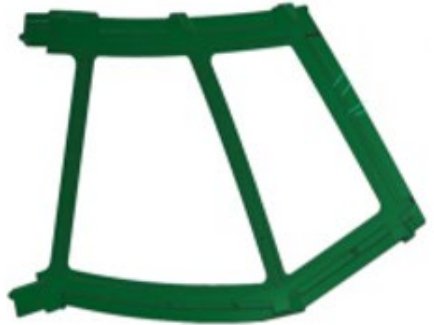

c)

Fig. 1. Examples of complex profiled, large parts of the airframe : a) spar; b) cross section; c) light frame.

It should be noted that any polymer-abrasive tool (petal circles, radial and end brushes) during processing is worn out and during continuous operation, adjustment of such a treatment mode as tool deformation is required. In practice, when machining the surfaces of parts (see Figure 1), which can be as heavy as $10 \mathrm{~m}$ or more, such a procedure is necessary due to a decrease in the productivity of the machining process, which is due to a reduction in tool deformation. Consider the nature of wear of the tool depending on the processing modes and the method of correcting its deformation $\Delta Y$ when treating the tops with elastic polymer-abrasive circles.

\section{Correction of deformation of elastic polymer-abrasive circles due to their wear when machining surfaces}

Research was conducted on the Deckel Maho DMC 635V universal milling machine on the simple samples representing plates with sizes 3 × 20 x $100 \mathrm{~mm}$ from V95pchT2 aluminum alloy.

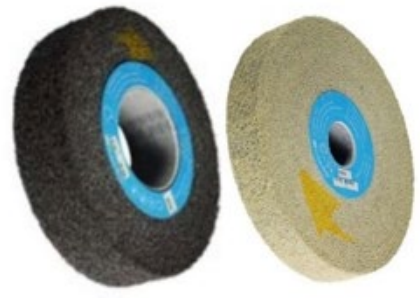

a)

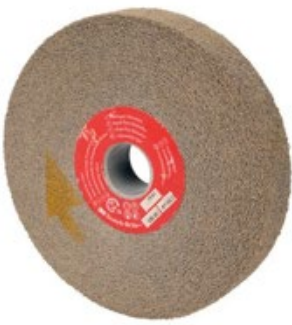

b)

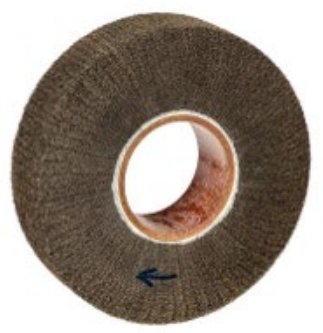

c)

Fig. 2. Elastic polymer-abrasive circles of company $3 M$ :

a) pressed circles of brand $F S-W L$; b) pressed circle of brand $D B-W L$; $)$ flap circle of brand $C F-F B$

The treatment was carried out with elastic polymer-abrasive circles of types FS-WL 8A MED, FS-WL 6S FIN, FS-WL 2S CRS, DB-WL 8S MED and CF-FB 0,5A FIN of company 3M (Minnesota Mining and Manufacturing Company) [8] (Figure 2), made of abrasive material Scotch-Brite ${ }^{\mathrm{TM}}$. This material consists of synthetic fibers forming a three-dimensional flexible 
nonwoven canvas throughout which abrasive grains are uniformly distributed. The main physical and mechanical data of the tools are given in Table 1. The work [9] is devoted to detailed research of physical and mechanical properties.

Table 1. Parameters of elastic polymer-abrasive circles

\begin{tabular}{|l|c|c|c|c|c|c|c|c|}
\hline \multicolumn{1}{|c|}{ Circle } & $\begin{array}{c}\boldsymbol{D}_{\boldsymbol{k}}, \\
\mathbf{m m}\end{array}$ & $\begin{array}{c}\boldsymbol{B}_{\boldsymbol{k}}, \\
\mathbf{m m}\end{array}$ & $\begin{array}{c}\boldsymbol{r}_{\boldsymbol{k}}, \\
\mathbf{m m}\end{array}$ & $\begin{array}{c}\boldsymbol{d}_{\boldsymbol{k}}, \\
\mathbf{m m}\end{array}$ & $\begin{array}{c}\boldsymbol{M}_{\boldsymbol{K}}, \\
\mathbf{k g}\end{array}$ & $\begin{array}{c}\boldsymbol{\gamma}_{\boldsymbol{\kappa}}, \\
\mathbf{k g} / \mathbf{m}^{\mathbf{3}}\end{array}$ & Abrasive & $\begin{array}{c}\text { Granularity } \\
\boldsymbol{Z}, \mathbf{m k m}\end{array}$ \\
\hline FS-WL-8AMED & 140,5 & 26 & 17,5 & 25,4 & 0,278 & 712,77 & $\mathrm{Al}_{2} \mathrm{O}_{3}$, & $50-60$ \\
\hline FS-WL-6SFIN & 129,5 & 25,5 & 17,5 & 25,4 & 0,162 & 501,63 & $\mathrm{SiC}$ & $45-50$ \\
\hline FS-WL-2SCRS & 147,2 & 26 & 17,5 & 25,4 & 0,162 & 377,37 & $\mathrm{SiC}$ & $\sim 100$ \\
\hline DB-WL-8SMED & 147,8 & 25,6 & 17,5 & 25,4 & 0,284 & 666,29 & $\mathrm{SiC}$ & $50-60$ \\
\hline CF-FB-0,5AFIN & 193 & 50 & 45 & 76,5 & 0,418 & 339,03 & $\mathrm{Al}_{2} \mathrm{O}_{3}$, & $45-50$ \\
\hline
\end{tabular}

$D_{k}$ - circle diameter, $\mathrm{mm} ; B_{k}-$ circle width, $\mathrm{mm} ; r_{k}$ - radius of circle bushing, $\mathrm{mm} ; d_{k}-$ diameter of hole, $\mathrm{mm} ; \quad M_{K}-$ circle weight, $\mathrm{kg} ; \gamma_{\kappa}$ - density of circle's material, $\mathrm{kg} / \mathrm{m}^{3}$.

The circle wear was determined by weighing it before and after processing on the analytical scales of the Ohaus series Discovery (DV) model DV214C. Wear value I ( $\mathrm{mm} / \mathrm{min})$ per unit time is used as circle wear index.

Value $I$ was calculated by the formula:

$$
I=\frac{G_{1}-G_{2}}{\gamma_{k} \cdot \pi \cdot D_{k} \cdot B \cdot T}
$$

where $\gamma_{k}$ - density of circle's material in $\mathrm{g} / \mathrm{mm}^{3}$ (see Table 1 ); $G$ and $G_{2}$ - circle weight before and after treatment, respectively, in g; $B$ - width of the sample to be treated, in $\mathrm{mm} ; D_{K}$ - circle diameter, in $\mathrm{mm}$ (see Table 1); $T$ - processing time of sample length 1 , in min.

The conclusion on the significance or insignificance of the influence of deformation of the circle of $\Delta Y$, velocity $\mathrm{V}$ and the supply of $\mathrm{S}$ on the wear of tool I was obtained using the Fisher criterion according to [10]. $\mathrm{F}_{0,95}$ is Fisher's criterion at a fiducial probability of 0.95 . This criterion at degrees of freedom $f_{1}=k-1=3$ and $f_{2}=k(n-1)=8$ according to reference data [10] is equal to $\mathrm{F}_{0,95}=4,1$. It is established that the circle wear does not depend on the feed $\mathrm{S}$.

Figure 3, $a, b$ shows the dependencies of circle wear for 1 minute of operation on the deformation of circles.

It is stated that the wear of the tool has been found to increase with increasing deformation. This is due to the fact that the vertical component of the cutting force [11] increases with increasing deformation, and therefore the friction force also increases.

Figure 4, $a, b$ shows the dependencies of circle wear in 1 minute of operation on the cutting speed. It has been established that tool wear is increasing with increasing speed.

This is due to the fact that with an increase in speed, the centrifugal and din components of the force of interaction of the abrasive grain along the treated surface grow. At the moment of meeting of abrasive grains of circle with treated surface impact occurs, as a result of which force becomes significantly more static [11]. At the same time, the centrifugal force and the impact pulse directly depend on the speed.

The experimental dependencies obtained were approximated and the following regression equation (wear $I$ in $\mathrm{mm} / \mathrm{min}$ ) was obtained: 


$$
I=a_{1} \cdot V^{2}+a_{2} \cdot \Delta Y^{2}+a_{3} \cdot V+a_{4} \cdot \Delta Y+a_{5} \cdot V \cdot \Delta Y+a_{6}
$$

where $V$ - cutting velocity, $\mathrm{m} / \mathrm{min} ; \Delta Y$ - circle deformation, $\mathrm{mm}$.

The values of the coefficients $a_{1-5}$ and the absolute terms $a_{6}$ of this equation for each of the instruments studied are shown in Table 2.

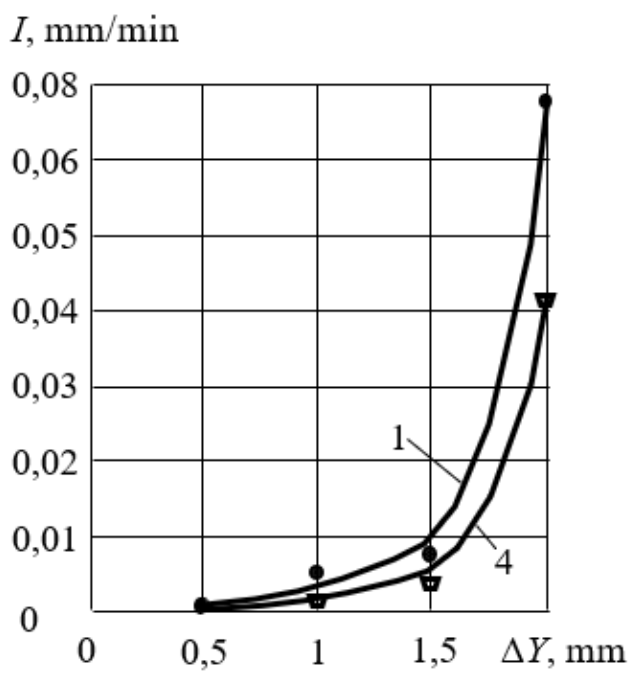

a)

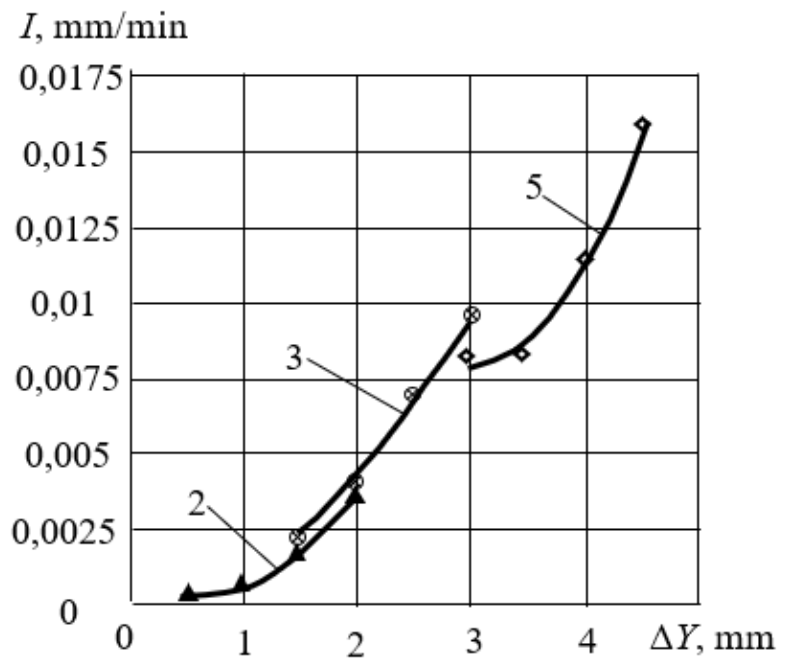

b)

Fig. 3. Dependency of wear for 1 min of operation from deformation $\triangle Y$ for circles: $1-F S$-WL $8 A$ MED at $V=441,4 \mathrm{~m} / \mathrm{min} ; 2-F S$-WL $6 S$ FIN at $V=406,8 \mathrm{~m} / \mathrm{min}$; $3-F S$-WL $2 S C R S$ at $V=462,4 \mathrm{~m} / \mathrm{min} ; 4-D B-W L 8 S M E D$ at $V=464,3 \mathrm{~m} / \mathrm{min}$; $5-C F-F B$ 0,5A FIN at $V=606,3 \mathrm{~m} / \mathrm{min}$.

\section{$I, \mathrm{~mm} / \mathrm{min}$}

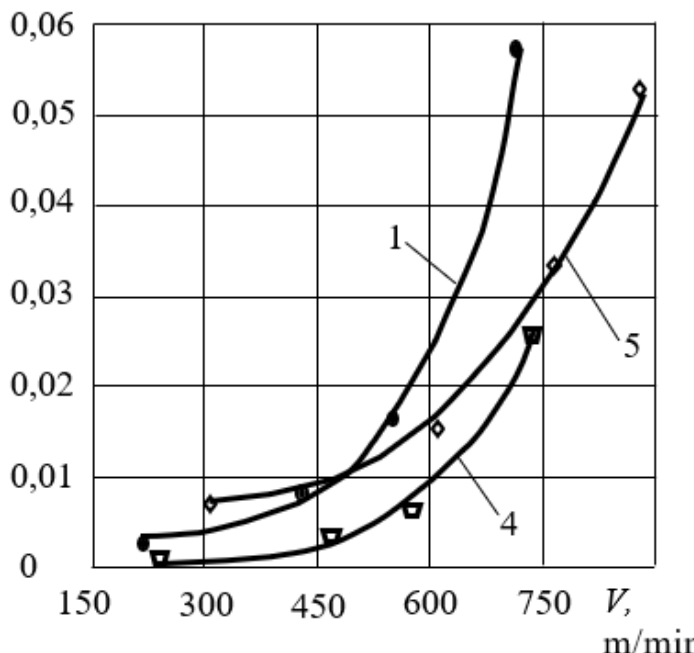

$I, \mathrm{~mm} / \mathrm{min}$

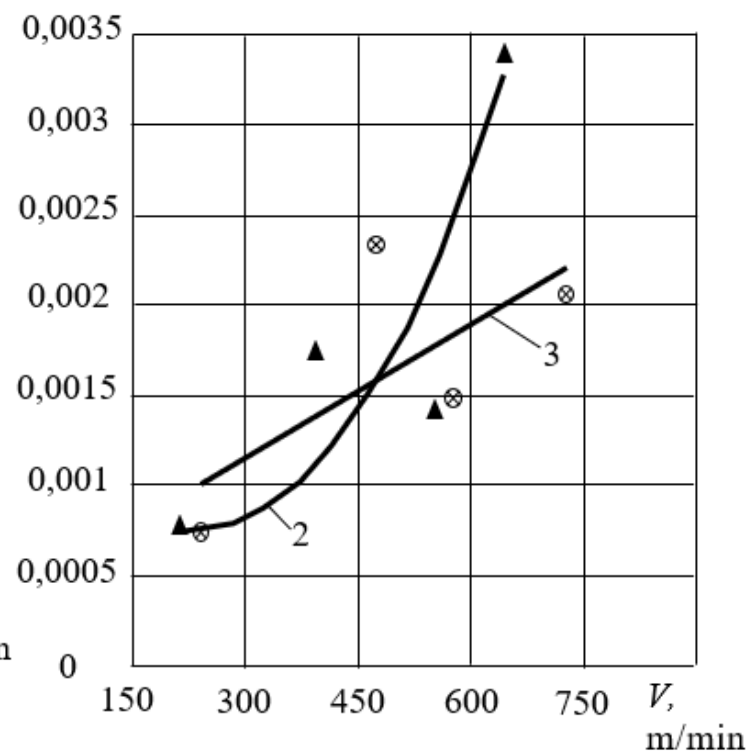

b)

Fig. 4. Dependency of instrument's wear for 1 min of operation from velocity of cutting $V$ for circles: 1 -FS-WL 8A MED; 2 - FS-WL 6S FIN; 3 -FS-WL 2S CRS; 4 -DB-WL 8S MED at deformation $\triangle Y=1,5 \mathrm{~mm} ; 5-C F-F B 0,5 A F I N$ at deformation $\triangle Y=4,5 \mathrm{~mm}$. 
Table 2. Values of coefficients and absolute terms in the equation (2)

\begin{tabular}{|c|c|c|c|c|c|}
\hline $\begin{array}{c}\text { Coeffi- } \\
\text { cient }\end{array}$ & $\begin{array}{c}\text { FS-WL 8A } \\
\text { MED }\end{array}$ & $\begin{array}{c}\text { FS-WL 6S } \\
\text { FIN }\end{array}$ & $\begin{array}{c}\text { FS-WL 2S } \\
\text { CRS }\end{array}$ & $\begin{array}{c}\text { DB-WL 8S } \\
\text { MED }\end{array}$ & $\begin{array}{c}\text { CF-FB 0,5A } \\
\text { FIN }\end{array}$ \\
\hline \hline $\boldsymbol{a}_{\boldsymbol{1}}$ & $6,3929 \cdot 10^{-8}$ & $-8,34 \cdot 10^{-10}$ & $-5,894 \cdot 10^{-9}$ & $8,869 \cdot 10^{-9}$ & $2,6883 \cdot 10^{-8}$ \\
\hline $\boldsymbol{a}_{\boldsymbol{2}}$ & $-4,16136 \cdot 10^{-4}$ & $4,2595 \cdot 10^{-4}$ & $1,3418328 \cdot 10^{-3}$ & $-5,78041 \cdot 10^{-7}$ & $1,089303 \cdot 10^{-3}$ \\
\hline $\boldsymbol{a}_{3}$ & $-6,2725 \cdot 10^{-6}$ & $2,62655 \cdot 10^{-6}$ & $-1,65935 \cdot 10^{-6}$ & $-9,2 \cdot 10^{-10}$ & $-3 \cdot 10^{-5}$ \\
\hline $\boldsymbol{a}_{4}$ & $9,07143 \cdot 10^{-3}$ & $-1,133675 \cdot 10^{-4}$ & $-4,264372 \cdot 10^{-3}$ & $8,843099 \cdot 10^{-3}$ & $-6,745733 \cdot 10^{-3}$ \\
\hline $\boldsymbol{a}_{\boldsymbol{5}}$ & $6,95575 \cdot 10^{-6}$ & $9,94751 \cdot 10^{-7}$ & $6,987568 \cdot 10^{-6}$ & $1,183662 \cdot 10^{-6}$ & $7,964633 \cdot 10^{-6}$ \\
\hline $\boldsymbol{a}_{\boldsymbol{6}}$ & $-6,91871 \cdot 10^{-3}$ & $-6,408575 \cdot 10^{-4}$ & $3,15588 \cdot 10^{-3}$ & $-1,668018 \cdot 10^{-3}$ & 0,01203567 \\
\hline
\end{tabular}

It should be noted that due to wear of the tool, the deformation of the circle $\Delta Y$ is gradually reduced, as a result of which the intensity of removal of the material decreases. Therefore, with a long processing process, it is necessary to periodically correct the deformation of the tool by the value of the worn out part of the $l_{i z n}$.

$$
l_{i z n}=I \cdot T_{i z n}
$$

where $T_{\mathrm{izn}}$ - time of treatment during which the wear $l_{\text {izn }}$ occurs, min;

$I$ - wear intensity which is calculated by formula (2), $\mathrm{mm} / \mathrm{min}$.

For the studied circles, the $T_{i z n}$ time (at the given allowable $l_{\text {izn }}$ values) is given in Table 3 (as an example) for the smallest $I_{\min }(\mathrm{mm} / \mathrm{min})$ and the highest $I_{\max }(\mathrm{mm} / \mathrm{min})$ of the possible wear intensity.

Table 3. Estimated time of the circle operation before correction of instrument tensioning $\Delta Y$

\begin{tabular}{|c|c|c|c|c|c|c|c|}
\hline \multirow{3}{*}{ Circle Type } & \multicolumn{2}{|c|}{ Wear I by (2) } & \multicolumn{5}{|c|}{ Wear lizn, mm } \\
\hline & \multirow{2}{*}{$\begin{array}{c}I_{\min }, \\
\mathrm{mm} / \mathrm{min}\end{array}$} & \multirow{2}{*}{$\begin{array}{c}I_{\max }, \\
\mathrm{mm} / \min \end{array}$} & 0,20 & 0,35 & 0,5 & 0,75 & 1,0 \\
\hline & & & \multicolumn{5}{|c|}{ Time of treatment $T_{\mathrm{izn}}, \mathrm{min}$} \\
\hline \multirow{2}{*}{ FS-WL 8A MED } & 0,002 & & 100 & 175 & 250 & 375 & 500 \\
\hline & & 0,078 & 2,6 & 4,5 & 6,4 & 9,6 & 12,8 \\
\hline \multirow{2}{*}{ FS-WL 6S FIN } & 0,0005 & & 400 & 700 & 1000 & 1500 & 2000 \\
\hline & & 0,0037 & 54,1 & 94,6 & 135,1 & 202,7 & 270,3 \\
\hline \multirow{2}{*}{ DB-WL 8S MED } & 0,002 & & 100 & 175 & 250 & 375 & 500 \\
\hline & & 0,041 & 4,9 & 8,5 & 12,2 & 18 & 24,4 \\
\hline \multirow{2}{*}{ FS-WL 2S CRS } & 0,002 & & 100 & 175 & 250 & 375 & 500 \\
\hline & & 0,01 & 20 & 35 & 50 & 75 & 100 \\
\hline \multirow{2}{*}{ CF-FB 0,5A FIN } & 0,0076 & & 26,3 & 46,1 & 65,8 & 98,7 & 131,6 \\
\hline & & 0,016 & 12,5 & 21,9 & 31,2 & 46,9 & 62,5 \\
\hline
\end{tabular}

\section{Conclusion}

The proposed method of correction of the $\Delta Y$ treatment mode during long-term surface treatment with elastic polymer-abrasive circles ensures effective control of the finishing process as the tool is worn. 


\section{References}

[1] Fomin A.A., Gusev V.G., Sadrtdinov A.R., Assurance of accuracy of longitudinal section of profile surfaces milled at high feeds, Lecture Notes in Mechanical Engineering. Part F4 (2019) 527-536. https://doi.org/10.1007/978-3-319-95630-5_55

[2] Fomin A.A., Gusev V.G., Timerbaev N.F., The processing of the profile surface of the work-pieces, characterized by low rigidity, Solid State Phenomena. 299 SSP (2020) 852-860. https://doi.org/10.4028/www.scientific.net/SSP.299.852

[3] Batuev V.A., Guzeev V.I., Batuev V.V., Determining the cut thickness in milling complex surfaces, Russian Engineering Research. 4 (2020) 343-346.

https://doi.org/10.3103/S1068798X20040061

[4] Tamarkin M., Tishchenko E., Chukarina I., Sosnitskaya T., Parts processing technology for transport engineering, Advanced in intelligent systems and computing. 1115 AISC (2020) 913922. https://doi.org/10.1007/978-3-030-37916-2_90

[5] Roshchupkin S.I., Sidorov D.E., Kolesov A.G., Assessment of the actual depth of cutting with cylindrical external grinding, IOP Conference Series: Materials Science and Engineering. International Conference on Modern Trends in Manufacturing Technologies and Equipment 2019, ICMTME 2019. Art. No. 022010 (2020). https://doi.org/10.1088/1757$899 \mathrm{X} / 709 / 2 / 022010$

[6] Makarov V.F., Muratov K.R., Gashev E.A., Optimal cutting time and speed in abrasive lapping of ceramic, Russian Engineering Research. 10 (2017) 916-918. https://doi.org/10.3103/S1068798X17100148

[7] Starodubtseva D.A., Koltsov V.P., Vinh L.T., Grinding of aluminum alloy panels after shot peen forming on contact type installations, IOP Conference Series: Materials Science and Engineering. 2019 International Conference on Innovations in Automotive and Aerospace Engineering, ICI2AE 2019. Art. No. 012109 (2019). https://doi.org/10.1088/1757$899 \mathrm{X} / 632 / 1 / 012109$

[8] Information on http://www.3mabrazives.ru/

[9] Dimov Yu. V., Podashev D.B., Properties of elastic abrasive wheels, Russian Engineering Research. 7 (2017) 631-637. https://doi.org/10.3103/S1068798X17070085

[10] Pystulnik E.I., Statistical methods of analysis and processing of observations, Nauka, Moscow, 1968.

[11] Dimov Yu. V., Podashev D.B., Cutting forces when machined with elastic abrasive circles, The messenger of Irkutsk National Research Technical University. 7 (2015) 47-55. 